\title{
Unternehmensmitbestimmung für Auslandsgesellschaften
}

\author{
Inhaltsübersicht
}
A. Einführung und Problemstellung
B. Paritätische Mitbestimmung in der Kritik
C. Flucht aus der Mitbestimmung durch Rechtsform-Shopping
I. Rechtstatsachen
II. Rechtsdogmatische Grundlagen des Rechtsform-Shopping
1. Mehrebenensystem des IPR
2. Europäisches Gesellschafts- kollisionsrecht
3. Völkerrechtliches Gesellschafts- kollisionsrecht
4. Autonomes deutsches Gesellschafts- kollisionsrecht

III. Rechtspolitische Reaktionen: Mitbestimmungs-Erstreckungsgesetz

D. Unternehmensmitbestimmung für Auslandsgesellschaften aus Sicht des IPR

I. Unternehmensmitbestimmung via Gesellschaftsstatut

II. Unternehmensmitbestimmung via Eingriffsstatut

1. Lehre von der Sonderanknüpfung international zwingender Normen
a) Wilhelm Wengler
b) Abgrenzung zum ordre public

2. Sonderanknüpfung im Gesellschaftsrecht

3. Allgemeine Voraussetzungen einer Sonderanknüpfung

4. Sonderanknüpfung des MitbestG 1976

E. Unternehmensmitbestimmung für Auslandsgesellschaften aus Sicht des Europarechts

I. Europarechtskonformität der Sonderanknüpfung?

II. Mitbestimmungs-Erstreckungsgesetz als Beschränkung der Niederlassungsfreiheit

III. Rechtfertigung der Anwendung der Unternehmensmitbestimmung auf Auslandsgesellschaften

1. Zwingender Grund des Allgemeininteresses

2. Geeignetheit

3. Erforderlichkeit
a) Betriebliche Mitbestimmung als Alternative?
b) Notwendigkeit einer Subsidiari- tätsklausel?
c) Unternehmensmitbestimmung in monistischen Auslandsgesell- schaften

F. Zusammenfassung

\section{A. Einführung und Problemstellung}

Unvergessen sind die vom Jubilar mit der ihn auszeichnenden Verve gehaltenen hochkarätigen gesellschafts- und bürgerlich-rechtlichen Vorlesungen und Seminare, die der Verf. während seiner Studienzeit an der Universität Heidelberg hören durfte und die ihm bis heute leuchtendes Vorbild engagiert-inter-

* Der Verf. dankt Frau Wiss. Mit. Marietta Pietrek, Freiburg, für die wertvolle Hilfe bei der Erstellung des Manuskriptes. 
aktiver Lehre sind. Ein brisantes Themenfeld, mit dem sich der Jubilar schon lange in Forschung und Lehre auseinandersetzt, ist die deutsche Unternehmensmitbestimmung. ${ }^{1}$ Gegenwärtig sind 681 Gesellschaften paritätisch mitbestimmt, für weitere ca. 1500 Gesellschaften gilt das Drittelbeteiligungsgesetz. ${ }^{2}$ An der Mitbestimmung scheiden sich indes die Geister: Während die einen sie kritisieren, ${ }^{3}$ betonen andere - gerade in Zeiten der Finanz- und Wirtschaftskrise - ihre Stärken ${ }^{4}$ (dazu unter B.). Fakt ist jedenfalls, dass immer mehr inländische Unternehmen sie offenbar als "belastend" empfinden und daher Umgehungsstrategien einschlagen: Sie schlüpfen in die Rechtsform einer Auslandsgesellschaft, ${ }^{5}$ was seit der durch die EuGH-Trilogie Centros, Überseering und Inspire Art geschaffenen Freiheit, Rechtsform-Shopping innerhalb der Europäischen Union zu betreiben, möglich ist. Vom Jubilar wurde diese Freiheit prägnant als „Europäische Rechtsformwahlfreiheit ${ }^{\prime \prime 6}$ gekennzeichnet (dazu unter C.).

Hinter der Nutzung einer Auslandsrechtsform für Inlandsaktivitäten steht das Kalkül, dass sich $\S 1$ MitbestG 1976 nicht auf ausländische Gesellschaftstypen erstreckt, weil sich sein Anwendungsbereich seinem Wortlaut nach auf die (jeweils deutsche) AG, KGaA, GmbH und eG bzw. nach § 4 MitbestG auf Kommanditgesellschaften mit einer deutschen Komplementärgesellschaft beschränkt. ${ }^{7}$ In der Tat meint die herrschende Meinung unter Bezug auf den Wortlaut des $\S 1$ MitbestG, mit Hilfe einer Auslandsgesellschaft könne man der deutschen Unternehmensmitbestimmung entgehen. ${ }^{8}$

1 Hommelhoff, Unternehmensführung in der mitbestimmten GmbH, ZGR 1978, $119 \mathrm{ff}$.; ders., Vereinbarte Mitbestimmung, ZHR 148 (1984), 118 ff.; ders., Arbeitnehmer-Beteiligung in der Europäischen Privatgesellschaft (SPE), GmbHR 2008, 1193 ff.; ders., Mitbestimmungsvereinbarungen zur Modernisierung der deutschen Unternehmensmitbestimmung - zum Gesetzentwurf des Arbeitskreises „Unternehmerische Mitbestimmung", ZGR 2010, $48 \mathrm{ff}$.

2 Sick, GmbHR 2011, 1196, 1197.

3 S. z.B. Ulmer, ZHR $166(2002), 271$ ff.

4 S. z.B. Sick, GmbHR 2011, 1196 ff.

5 Näher unter C.I.

6 Der Verf. hatte die Ehre, seine Doktorarbeit unter Betreuung des Jubilars anfertigen zu dürfen. Der Jubilar entwickelte dabei im Rahmen einer der Promotionsbetreuungsgespräche den treffenden Titel „Europäische Rechtsformwahlfreiheit", den der Verf. gerne übernommen hat: Weller, Europäische Rechtsformwahlfreiheit und Gesellschafterhaftung, 2004.

7 Nach $\S 4$ MitbestG erstreckt sich die Unternehmensmitbestimmung ferner auf gewisse Gesellschaftstypenkombinationen, insbesondere auf die GmbH \& Co. KG sowie die AG \& Co. KG.

8 So etwa Eberspächer, ZIP 2008, 1951, 1958; Ebke, IZ 2003, 927, 930; ders., BB 2003, Heft 1, S. I: Die unternehmerische Mitbestimmung lasse sich „wohl kaum" gegen ein fremdes Gesellschaftsstatut durchsetzen; Henssler in GS Heinze, 2005, S. 333, 341 f.; Merkt, ZIP 2011, 1237, 1239; Teichmann, ZIP 2009, 1787, 1787; Ulmer, JZ 1999, 662, 663; Zimmer, NIW 2003, 3585, 3590. Vgl. auch das obiter dictum in BGH, Vorlagebeschluss v. 30.3.2000 - VII ZR 370/98, ZIP 2000, 967, 968 - Überseering: „Beispielsweise ist nicht erkennbar, wie die unternehmerische Mitbestimmung des deutschen Rechts in einer ausländischen Gesellschaft verwirklicht werden soll, die keinen Aufsichtsrat hat." 
Ob diese Argumentation belastbar ist, erscheint indes zweifelhaft. ${ }^{9}$ Denn naturgemäß sind die Sachnormen des deutschen Rechts auf den Inlandssachverhalt als typische Fallkonstellation zugeschnitten, ${ }^{10}$ so dass es nicht überrascht, dass der Wortlaut des $\S 1$ MitbestG nur deutsche Rechtsformen adressiert. Indes soll hier nicht der Frage nachgegangen werden, ob das MitbestG 1976 schon nach der lex lata auf Auslandsgesellschaften anzuwenden ist, was qua richterrechtlicher Sonderanknüpfung denkbar wäre. ${ }^{11}$ Denn angesichts der mit einer richterrechtlichen Sonderanknüpfung einhergehenden Rechtsunsicherheit scheut sich die Arbeitnehmerseite offenbar, die Mitbestimmung bei Auslandsgesellschaften gerichtlich durchzusetzen. Die gesellschafterseitige Gestaltungspraxis nutzt diese Passivität und orientiert sich an der vorgenannten herrschenden Meinung mit der Konsequenz, dass die Unternehmensmitbestimmung momentan de facto auf Auslandsgesellschaften mit Verwaltungssitz im Inland keine Anwendung findet. ${ }^{12}$

Vor diesem Hintergrund soll im Folgenden der höchst virulenten Frage nachgegangen werden, inwieweit das deutsche Mitbestimmungsregime de lege ferenda qua Legislativakt auf Auslandsgesellschaften erstreckt werden kann. ${ }^{13}$ Diskutiert wird insbesondere ein Mitbestimmungs-Erstreckungsgesetz, ${ }^{14}$ wel- $^{13}$ ches die Anwendbarkeit der deutschen Unternehmensmitbestimmung auf Auslandsgesellschaften explizit anordnen und damit die vorgenannte Unsicherheit einer richterrechtlichen Erstreckung des gegenwärtigen Mitbestimmungsregimes beseitigen soll. Die Wettbewerbsgleichheit zwischen in- und ausländischen Gesellschaften streitet für ein solches Gesetz. ${ }^{15}$ Aus Sicht der Rechtsdogmatik stellt sich dabei die Frage, ob sich ein solches Gesetz systemkohärent legitimieren ließe. Die Antwort ist dem auch vom Jubilar immer wieder ausgemessenen Spannungsfeld zwischen Gesellschaftsrecht, IPR und Europarecht $\mathrm{zu}$ entnehmen. ${ }^{16}$ Dabei ist $\mathrm{zu}$ trennen zwischen einer Systemkohärenz aus Sicht des IPR (dazu unter D.) und derjenigen aus Sicht des Europarechts (dazu unter E.).

9 Ähnlich skeptisch Kindler, NJW 2003, 1073, 1079; Thüsing, ZIP 2004, 381.

10 v. Bar/Mankowski, IPR, Bd. 1, 2. Aufl. 2003, § 4 Rz. 17. Dies vernachlässigt z. B. Schall, NJW 2011, $3745 \mathrm{ff}$.

11 Hierzu Weller in MünchKomm. GmbHG, 2010, Int. GesR, Einl. Rz. 474 ff.

12 Sick, GmbHR 2011, 1196, 1197.

13 Hierzu zuletzt Merkt, ZIP 2011, 1237 ff.; Hübner, Kollisionsrechtliche Behandlung von Gesellschaften aus „nicht-privilegierten“ Drittstaaten, 2010, S. 239 ff.; s. auch Hommelhoff, ZGR 2010, 48, 55

14 S. infra unter C.III.

15 Hommelhoff, ZGR 2010, 48, 55, der sein Petitum eines Einbezugs ausländischer Gesellschaftsformen freilich verbindet mit einer Öffnung der Unternehmensmitbestimmung für privatautonome Mitbestimmungsvereinbarungen (S. 56 ff.).

16 Vgl. etwa Hommelhoff, Zivilrecht unter dem Einfluss europäischer Rechtsangleichung, AcP 192 (1992), 71 ff.; ders., Europäisierung und Internationalisierung des Gesellschafts- und Unternehmensrechts in Deutschland, in Müller-Graff/Roth (Hrsg.), Recht und Rechtswissenschaft, 2001, S. 133 ff. 


\section{B. Paritätische Mitbestimmung in der Kritik}

Die paritätische Unternehmensmitbestimmung, wie sie das MitbestG 1976 für Unternehmen mit mehr als 2.000 Arbeitnehmern vorschreibt, steht in mehrfacher Hinsicht in der Kritik. ${ }^{17}$ Deutschland ist das einzige Land mit marktwirtschaftlicher Wirtschaftsordnung, welches eine paritätische Unternehmensmitbestimmung kennt; ${ }^{18}$ sie gilt vor diesem Hintergrund als Wettbewerbsnachteil für international tätige deutsche Unternehmen. ${ }^{19}$ Kritiker monieren ferner, sie beeinträchtige die Kontrollfunktion des Aufsichtsrates, weil dieser aufgrund der Arbeitnehmerbeteiligung primär die Interessen der stakeholder vertrete und daher zu viel Zeit für Personal- und Sozialfragen aufwende. ${ }^{20}$ Dies führe zu negativen Begleiterscheinungen in Gestalt einer inhaltlichen Entleerung der Diskussionen im Aufsichtsratsplenum und zu einer Diskussionsverlagerung in Ausschüsse sowie zur Ausdünnung der Zustimmungskataloge nach $\S 111$ Abs. 4 Satz 2 AktG. ${ }^{21}$ Schließlich sei sie bei Strukturmaßnahmen oder Umwandlungen häufig ein Hemmnis für den Informationsfluss zwischen Vorstand und Aufsichtsrat, fürchte doch der Vorstand bei solchen Maßnahmen den Widerstand der Arbeitnehmervertreter. ${ }^{22}$

Bei legislativen Vorhaben erweist sich die Unternehmensmitbestimmung mitunter ebenfalls als "Bremsklotz": Die Verabschiedung eines Statuts für die Societas Europaea scheiterte lange an der Mitbestimmungsfrage; nach mehrjährigen Verhandlungen brachte hier erst die sog. Verhandlungslösung den Durchbruch. ${ }^{23}$ Dagegen ist die Regelung der Mitbestimmung bei der Societas Privata Europaea (SPE) nach wie vor umstritten und einer der wesentlichen Gründe, weshalb die Einführung dieser Rechtsform für den Mittelstand bislang scheiterte. ${ }^{24}$ Die Mitbestimmung soll schließlich der Grund dafür sein, weshalb die Kodifikation des Internationalen Gesellschaftsrechts im EGBGB ${ }^{25}$ nicht weiter verfolgt wird: Durch die im Referentenentwurf 2008 vorgesehene Statuierung der Gründungstheorie als allseitige autonome gesellschaftsrecht-

17 Statt vieler Ulmer, ZHR 166 (2002), 271 ff.; ferner Hübner, Kollisionsrechtliche Behandlung von Gesellschaften aus "nicht-privilegierten“ Drittstaaten, 2010, S. $236 \mathrm{ff}$.

18 Rechtsvergleichender Überblick bei Hopt, ZHR 175 (2011), 444, 503 ff.

19 Ulmer, ZHR $166(2002), 271,274$

20 Ulmer, ZHR $166(2002), 271,276$.

21 Ulmer, ZHR $166(2002), 271,275$.

22 Hopt, ZGR 2000, 779, 801.

23 Teichmann, Binnenmarktkonformes Gesellschaftsrecht, 2006, S. 252.

24 Näher zu einem möglichen Mitbestimmungsmodell für die SPE Hommelhoff, GmbHR $2008,1193 \mathrm{ff}$.

25 Referentenentwurf für ein Gesetz zum Internationalen Privatrecht der Gesellschaften, Vereine und juristischen Personen v. 7.1.2008; hierzu Bollacher, RIW 2008, 200 ff.; Clausnitzer, NZG 2008, 321 ff.; Leuering, ZRP 2008, 73, 75 ff.; Köster, ZRP 2008, 214; Kußmaul/Richter/Ruiner, DB 2008, 451; Schneider, BB 2008, 566; Wagner/Timm, IPRax 2008, 81 ff. Der Referentenentwurf geht auf einen entsprechenden Vorschlag einer Spezialkommission "Internationales Gesellschaftsrecht" des Deutschen Rates für IPR zurück, vgl. Sonnenberger (Hrsg.), Vorschläge und Berichte zur Reform des europäischen und deutschen internationalen Gesellschaftsrechts, 2007. 
liche Kollisionsregel (vgl. Art. 10 Abs. 1 EGBGB-Entwurf ${ }^{26}$ ) könne - so wird befürchtet - die Mitbestimmung durch Einsatz ausländischer Rechtsformen mit inländischem Verwaltungssitz umgangen werden. ${ }^{27}$

Vor diesem Hintergrund überrascht es nicht, dass immer wieder rechtspolitische Vorschläge zur sachrechtlichen Reform der Unternehmensmitbestimmung unterbreitet werden, ${ }^{28}$ die meist auf eine Flexibilisierung des bisherigen Regimes durch Einräumung privatautonomer Gestaltungsmacht in Anlehnung an das Verhandlungsmodell bei der SE hinauslaufen. ${ }^{29}$

\section{Flucht aus der Mitbestimmung durch Rechtsform-Shopping}

\section{Rechtstatsachen}

In praxi wird die Unternehmensmitbestimmung häufig abzumildern oder durch „Rechtsform-Shopping ${ }^{\prime 30}$ ganz zu vermeiden gesucht. Rechtsform-Shopping ist aus inländischer Perspektive - wie nachfolgend unter C.II. skizziert werden soll - insbesondere in Bezug auf Gesellschaften aus anderen EU-Mitgliedstaaten, die der Gründungstheorie folgen, möglich, ebenso im Hinblick auf Gesellschaften aus den USA, denen gegenüber sich die Bundesrepublik Deutschland völkerrechtlich zur Anwendung der Gründungstheorie verpflichtet hat.

Nach einer Studie der Hans Böckler-Stiftung ${ }^{31}$ sollen zahlreiche Unternehmen bereits aus der Mitbestimmung „geflüchtet" sein, indem sie anstelle einer deutschen $\mathrm{GmbH}$ oder AG eine ausländische Rechtsform als Plattform für ihre

26 "Gesellschaften, Vereine und juristische Personen des Privatrechts unterliegen dem Recht des Staates, in dem sie in ein öffentliches Register eingetragen sind. Sind sie nicht oder noch nicht in ein öffentliches Register eingetragen, unterliegen sie dem Recht des Staates, nach dem sie organisiert sind."

27 Vgl. auch Weiß/Seifert, ZGR 2009, 542, 543 ff. sowie den Beschluss J 001 des 19. DGB-Bundeskongresses, der eine Mitbestimmungserstreckung fordert, da "für mitbestimmungsfeindliche Unternehmen Anreize [bestünden], durch eine entsprechende Konstruktion ihrer Rechtsform der deutschen Unternehmensmitbestimmung zu entgehen."

28 Baums/Bachmann/Habersack/Henssler/Lutter/Oetker/Ulmer, Entwurf einer Regelung zur Mitbestimmungsvereinbarung sowie zur Größe des mitbestimmten Aufsichtsrats, ZIP 2009, 885 ff.; hierzu Hommelhoff, Mitbestimmungsvereinbarungen zur Modernisierung der deutschen Unternehmensmitbestimmung - zum Gesetzentwurf des Arbeitskreises „Unternehmerische Mitbestimmung", ZGR 2010, 48 ff.; Teichmann, Beilage zu ZIP 48/2009, 10 ff.; vgl. ferner Arbeitskreis Aktien- und Kapitalmarktrecht, Vorschläge zur Reform der Mitbestimmung in der Societas Europaea (SE), ZIP 2010, $2221 \mathrm{ff}$.

29 Hommelhoff, ZGR 2010, 48, 52, 56 ff.

30 In Anlehnung an den internationalprivatrechtlichen Terminus des forum shopping, bei dem es um die gezielte Wahl von Gerichtsständen geht, kann bei der Wahl von Gesellschaftstypen von „Rechtsform-Shopping“ gesprochen werden.

31 Sick, Mitbestimmungsrelevante Unternehmen mit ausländischen/kombiniert ausländischen Rechtsformen, Studie der Hans Böckler-Stiftung, 2010; ders., GmbHR 2011, $1196 \mathrm{ff}$. 
inländische Unternehmenstätigkeit wählen ${ }^{32}$ oder aber die Auslandsgesellschaft als Komplementär einer (deutschen) KG einsetzen: So werde - Stand Mai 2011 die paritätische Mitbestimmung von 16 deutschen Personengesellschaften durch Implementierung eines ausländischen Komplementärs (insbesondere in der Form der Limited \& Co. $\mathrm{KG}^{33}$ ) vermieden. ${ }^{34}$ Als prominentes Beispiel gilt die Fluggesellschaft Air Berlin PLC \& Co. Luftverkehrs KG, deren Komplementär eine englische Public Limited Company (PLC) ist. ${ }^{35}$ Weitere bekannte Unternehmen sind die Drogeriemarktkette Müller Ltd. \& Co. KG, das Logistikunternehmen Dachser GmbH (Österreich) \& Co. KG, der Druckkonzern Privonis Ltd. \& Co. $\mathrm{KG}^{36}$ und seit Kurzem das Modeunternehmen Zara B.V. \& Co. KG sowie das Berliner Recyclingunternehmen ALBA PLC \& Co. KG. ${ }^{37}$ Die Tochter des schwedischen Textilkonzerns H \& $M$ soll genau in dem Moment ihre Rechtsform von einer $\mathrm{GmbH}$ in eine B.V. \& Co. KG geändert haben, als die Arbeitnehmer einen mitbestimmten Aufsichtsrat forderten. ${ }^{38}$

\section{Rechtsdogmatische Grundlagen des Rechtsform-Shopping}

Die Anerkennung ${ }^{39}$ ausländischer Gesellschaften im Inland und damit ihr Einsatz als potentielles Vehikel zur Vermeidung der Mitbestimmung hängen von der Anknüpfung des Gesellschaftsstatuts ab. ${ }^{40}$ Als Faustformel gilt: Nur wenn das IPR der lex fori das Gründungsrecht einer Gesellschaft zur Anwendung beruft, wird die ausländische Gesellschaft als solche anerkannt, d.h. als ausländische Gesellschaft und nicht lediglich im Sinne der sog. Wechselbalgtheorie als von einer ausländischen Kapital- in eine inländische Personengesellschaft umqualifizierte Rechtsform behandelt. ${ }^{41}$ Die Anknüpfung richtet

32 Die Deer \& Company Inc. und die McDonald's Deutschland Inc. sollen der paritätischen Mitbestimmung ausweichen, indem sie sich als US-amerikanische Kapitalgesellschaft organisieren, Sick, Mitbestimmungsrelevante Unternehmen mit ausländischen/kombiniert ausländischen Rechtsformen, 2010, S. 5.

33 Eingehend zu dieser Typenkombination Höhne, Die Ltd. \& Co KG, 2011.

34 Unter den ausländischen Rechtsformen ist die niederländische B.V. mit sechs Komplementärunternehmen am stärksten vertreten, gefolgt von fünf britischen Limiteds (Ltd. und PLC), Sick, Mitbestimmungsrelevante Unternehmen mit ausländischen/ kombiniert ausländischen Rechtsformen, 2010, S. 4 (Stand Nov. 2009); s. ergänzend Sick, GmbHR 2011, 1196, 1197 (Stand Mai 2011).

35 Angeblich soll der (frühere) CEO von Air Berlin, Joachim Hunold, geäußert haben, mit dieser grenzüberschreitenden Gesellschaftstypenkombination deutsche Gesetze zur Mitbestimmung umgehen zu wollen, vgl. BT-Drucks. 14/1713, S. 2; vgl. hierzu auch Hommelhoff, ZGR 2010, 48, 55.

36 Vgl. BT-Drucks. 17/1413, S. 2.

37 Sick, GmbHR 2011, 1196, 1197.

38 Sick, GmbHR 2011, 1196, 1197.

39 Zum Begriff der Anerkennung Kindler in MünchKomm. BGB, 5. Aufl. 2010, Int. GesR, $\mathrm{Rz} .316 \mathrm{ff}$.

40 Hierzu aus jüngerer Zeit etwa Hübner, Kollisionsrechtliche Behandlung von Gesellschaften aus "nicht-privilegierten" Drittstaaten, 2010, S. $39 \mathrm{ff}$.

41 Ausführlich zur Wechselbalgtheorie Weller in FS Goette, 2011, S. 583 ff. 
sich nach Kollisionsregeln, für welche die in Art. 3 EGBGB zum Ausdruck kommende Rechtsquellendogmatik des IPR maßgeblich ist. ${ }^{42}$

\section{Mehrebenensystem des IPR}

Nach Art. 3 EGBGB gibt es drei verschiedene Ebenen an Rechtsquellen, denen eine Kollisionsregel zur Bestimmung des Gesellschaftsstatuts entspringen kann:43 (1.) das Europarecht, (2.) das Völkerrecht, und (3.) das autonome deutsche Recht. Welche Rechtsquelle in diesem Mehrebenensystem jeweils einschlägig ist, hängt von ihrem Anwendungsbereich ab. Im Fall überlappender Anwendungsbereiche genießen die europarechtlichen Kollisionsregeln in der Normenhierarchie Vorrang gegenüber den völkerrechtlichen sowie gegenüber den autonomen deutschen Kollisionsregeln; dies ergibt sich aus dem unionsrechtlich fundierten Anwendungsvorrang des EU-Rechts. ${ }^{44}$

\section{Europäisches Gesellschaftskollisionsrecht}

Zwecks Umgehung der Mitbestimmung wird bislang hauptsächlich auf Rechtsformen aus anderen EU-Staaten zurückgegriffen. Deren Gesellschaftsstatut bestimmt sich nach dem europäischen Gesellschaftskollisionsrecht. Dieses gilt für Kapital- und Personenhandelsgesellschaften ${ }^{45}$ aus anderen EU-Mitgliedstaaten sowie für Gesellschaften aus den EWR-Staaten Liechtenstein, Norwegen und Island, ${ }^{46}$ nicht dagegen für Gesellschaften aus allen sonstigen Staaten, den sog. Drittstaaten. Seine Grundlage hat das europäische Gesellschaftskollisionsrecht in der Niederlassungsfreiheit nach Art. 49, 54 AEUV. Diese

42 Brödermann, NJW 2010, 807, 809, 813; Weller, IPRax 2009, 202, 204 ff.

$43 \mathrm{Vgl}$. allgemein zu den Rechtsquellen im IPR und ihrem Rangverhältnis v. Hoffmann/ Thorn, IPR, 9. Aufl. 2007, § 1 Rz. 72 ff.

44 Anders als Art. 3 Nr. 2 EGBGB hat Art. 3 Nr. 1 EGBGB lediglich eine deklaratorische Funktion. Denn der Anwendungsvorrang des EU-Kollisionsrechts folgt nicht aus dem nationalen Recht, sondern aus dem Unionsrecht selbst, v. Bar/Mankowski, IPR, Bd. 1, 2. Aufl. 2003, 33 Rz. 37. Näher zum Anwendungsvorrang des EU-Rechts Wiedmann in Gebauer/Wiedmann (Hrsg.), Zivilrecht unter europäischem Einfluss, 2. Aufl. 2010, Kapitel 2, Rz. 2 ff., 11 ff.

45 Personengesellschaften werden jedenfalls dann von der Niederlassungsfreiheit erfasst, wenn sie wirtschaftlich tätig sind (vgl. Art. 54 Abs. 2 AEUV: „Erwerbszweck verfolgen"), was bei Handelsgesellschaften gemäß $\S 105$ Abs. 1 i.V.m. $\S 1$ Abs. 2 HGB der Fall ist, vgl. auch Koch, ZHR 173 (2009), 101, 112 ff.

46 Die Niederlassungsfreiheit in Art. 31, 34 EWRV hat ebenfalls einen kollisionsrechtlichen Gehalt; sie beinhaltet für zuziehende Gesellschaften aus den EWR-Mitgliedstaaten Liechtenstein, Norwegen und Island gleichfalls die Gründungstheorie. Denn die Art. 31, 34 EWRV statuieren entsprechend der Zielsetzung des EWR-Vertrages, die Mitgliedstaaten des EWR in die Regelungen des EU-Binnenmarktes mit einzubeziehen, eine den Art. 49, 54 AEUV inhaltsgleiche Regelung zur Niederlassungsfreiheit. Dementsprechend gelten die EuGH-Entscheidungen Centros, Überseering und Inspire Art auch für die Interpretation der Niederlassungsfreiheit im EWR (Auslegungsgleichklang), BGH v. 19.9.2005 - II ZR 372/03, NTW 2005, 3351; näher Weller, ZGR 2006, 748 ff. 
enthält nach vorzugswürdiger Meinung ${ }^{47}$ für Gesellschaften, die nach der Rechtsordnung eines EU-Mitgliedstaats gegründet worden sind, zwar nicht für Wegzugskonstellationen (Daily Mail, Cartesio), sehr wohl aber für Zuzugskonstellationen (Centros, Überseering, Inspire Art) eine versteckte Kollisionsregel in Gestalt der Gründungstheorie. ${ }^{48}$ Der BGH ist jüngst in einer Entscheidung zu Art. 22 EuGVO$^{49}$ implizit ebenfalls von der Niederlassungsfreiheit als versteckter Kollisionsregel ausgegangen. ${ }^{50}$ Zur näheren Begründung der Ansicht von der Niederlassungsfreiheit als versteckter Kollisionsregel sei auf einen Beitrag verwiesen, der auf Anregung des Jubilars in der von ihm geschäftsführend verantworteten ZGR erschienen ist. ${ }^{51}$

Entnimmt man der Niederlassungsfreiheit eine Gründungsrechtsanknüpfung, werden etwa nach englischem Recht gegründete Gesellschaften mit Verwaltungssitz in Deutschland (wie die Air Berlin PLC) anerkannt: Über die in Art. 49, 54 AEUV (versteckt) verankerte Gründungstheorie gelangt man zum englischen Recht. Geht man dabei von einer Sachnormverweisung aus (Art. 3a Abs. 1 EGBGB), welche Kollisionsnormen kennzeichnet, die - wie die Gründungstheorie ${ }^{52}$ - Ausdruck der Rechtswahlfreiheit sind (vgl. Art. 4 Abs. 2 EGBGB), so wird unmittelbar auf das englische Gesellschaftsrecht verwiesen. Nimmt man dagegen eine Gesamtverweisung (Art. 4 Abs. 1 Satz 1 EGBGB) an, gelangt man zum englischen Kollisionsrecht. Da jenes der Gründungstheorie folgt, kommt es nicht $\mathrm{zu}$ einem renvoi auf das deutsche Recht (vgl. Art. 4 Abs. 1 Satz 2 EGBGB); vielmehr nimmt das englische IPR die Verweisung an. Nach beiden Ansätzen gelangt man mithin zum materiellen englischen Gesellschaftsrecht.

\section{Völkerrechtliches Gesellschaftskollisionsrecht}

Das völkerrechtliche Gesellschaftskollisionsrecht gilt für Gesellschaften aus Staaten außerhalb der EU, mit denen Deutschland in einem Staatsvertrag eine entsprechende Kollisionsregel vereinbart hat. ${ }^{53}$ Eine Paradebeispiel hierfür ist Art. XXV Abs. 5 Satz 2 Freundschafts-, Handels- und Schifffahrtsvertrag zwischen der Bundesrepublik Deutschland und den Vereinigten Staaten von

47 Eidenmüller, ZIP 2002, 2233, 2241; Weller, Europäische Rechtsformwahlfreiheit und Gesellschafterhaftung, 2004, S. 29 ff., 51 ff., 95 ff.; a. A. Kindler, IPRax 2009, 189, 191; Mansel, RabelsZ 2006, 651, 671 ff.: Die Niederlassungsfreiheit beinhalte eine Ergebnisvorgabe, der entweder in Form eines Rechtsanwendungsbefehls/Verweisung (in Gestalt der Gründungstheorie), einer verfahrensrechtlichen Anerkennung oder aber in Form eines Anerkennungsstatuts Rechnung getragen werden könne.

48 Zur Differenzierung zwischen Wegzugs- und Zuzugskonstellationen Weller, IPRax $2003,324,327 \mathrm{f}$.

49 BGH v. 12.7.2011 - II ZR 28/10, ZIP 2011, 1837 ff.

50 Thomale, NZG 2011, 1290.

51 Weller, ZGR 2010, 679, $696 \mathrm{ff}$

52 Die Gründungstheorie gilt als Spielart der Rechtswahlfreiheit, Jayme, Kollisionsrechtliche Parteiautonomie, in Jud/Rechberger (Hrsg.), Kollisionsrecht in der EU, 2008, S. 63,68 .

53 Überblick zu den Staatsverträgen mit gesellschaftskollisionsrechtlichem Gehalt bei Kindler in MünchKomm. BGB, 5. Aufl. 2010, Int. GesR Rz. 308 f. 
Amerika vom 29.10.1954. ${ }^{54}$ Diese Bestimmung enthält nach herrschender Ansicht eine - ebenfalls versteckte - Kollisionsregel in Form der Gründungstheorie für US-Gesellschaften. ${ }^{55}$

\section{Autonomes deutsches Gesellschaftskollisionsrecht}

Dagegen ist mit Gesellschaften aus sog. Drittstaaten, etwa der Schweiz, ein Rechtsform-Shopping zwecks Vermeidung der Mitbestimmung nicht sinnvoll. ${ }^{56}$ Denn Drittstaatengesellschaften mit Verwaltungssitz in Deutschland werden im Inland nicht als ausländische Gesellschaften anerkannt; sie unterstehen vielmehr nach der vom $\mathrm{BGH}^{57}$ vertretenen modifizierten Sitztheorie deutschem Sachrecht. Angesichts des im Inland geltenden numerus clausus an Rechtsformen werden sie - mangels Registereintragung im Inland - nicht als Kapitalgesellschaft, sondern als inländische Personengesellschaft /GbR oder OHG) behandelt. Aufgrund der Konsequenz der modifizierten Sitztheorie, der Umqualifizierung in eine inländische Gesellschaft, ist auch von der „Wechselbalgtheorie ${ }^{\prime 58}$ die Rede. Mit einer in eine inländische Personengesellschaft umqualifizierten Auslandsgesellschaft ließe sich zwar auch die Mitbestimmung vermeiden, weil § 1 MitbestG auf die GbR und OHG keine Anwendung findet. Allerdings sind die übrigen mit der Umqualifizierung einhergehenden sachrechtlichen Folgen so unattraktiv (z.B. Problematik der Selbstorganschaft bei Personengesellschaften sowie persönliche Gesellschafterhaftung gemäß $\S 128$ $\mathrm{HGB}^{59}$ ), dass Drittstaatengesellschaften als Vehikel der Rechtsgestaltung typischerweise ausscheiden.

\section{Rechtspolitische Reaktionen: Mitbestimmungs- Erstreckungsgesetz}

Die zunehmende „Flucht aus der Mitbestimmung" via Rechtsform-Shopping soll nach Ansicht verschiedener politischer Parteien gestoppt werden. Einem Antrag der SPD-Bundestagsfraktion ${ }^{60}$ zufolge sollen mitbestimmungsfreie

54 BGBl. II 1956, 488; hierzu Hübner (Fn. 13), S. 79 ff.; Kaulen, Die Anerkennung von Gesellschaften unter Art. XXV Abs. 5 S. 2 des deutsch-US-amerikanischen Freundschafts-, Handels- und Schifffahrtsvertrags von 1954, 2008; Tomschin, Die Anerkennung US-amerikanischer Gesellschaften in Deutschland, 2010.

55 BGH v. 13.10.2004 - I ZR 245/01 - GEDIOS, IPRax 2005, 340: „Das Personalstatut (Gesellschaftsstatut) einer juristischen Person und damit auch deren Rechts- und Parteifähigkeit im Verhältnis zwischen der Bundesrepublik Deutschland und den Vereinigten Staaten von Amerika richtet sich grundsätzlich nach dem Recht des Staates, in dem die juristische Person gegründet wurde. " Hierzu Stürner, IPRax 2005, 305; vgl. ferner BGH v. 29.1.2003 - VIII ZR 155/02, IPRax 2003, 265.

56 Drittstaaten sind Länder, die weder von der EU/EWR-Niederlassungsfreiheit profitieren, noch von einer völkervertraglich vereinbarten Gründungsrechtsanknüpfung.

57 BGHZ 178, 192, Tz. 21 - Trabrennbahn; hierzu Weller, IPRax 2009, 202 ff.

58 Weller in FS Goette, 2011, S. 583 ff.

59 Näher Weller in FS Goette, 2011, S. 583, 593 ff.

60 Antrag "Demokratische Teilhabe von Belegschaften und ihren Vertretern an unternehmerischen Entscheidungen stärken" v. 16.6.2010, BT-Drucks. 17/2122. 
Zonen für Unternehmen ausländischer Rechtsform verringert werden, damit (wieder) mehr Arbeitnehmerinnen und Arbeitnehmer in den Schutzbereich der Unternehmensmitbestimmung fallen. Dies soll erreicht werden, indem die deutsche Mitbestimmung durch eine ausdrückliche gesetzliche Regelung auf Unternehmen ausländischer Rechtsform mit Verwaltungssitz oder Zweigniederlassung in Deutschland bzw. deutsche Personengesellschaften mit ausländischem Komplementär erstreckt wird. ${ }^{61}$ Die Fraktion Die Linke fordert ebenfalls, „die gesetzlichen Voraussetzungen dafür zu schaffen, dass die deutschen Vorschriften über die unternehmerische Mitbestimmung - namentlich die des Drittelbeteiligungsgesetzes und des Mitbestimmungsgesetzes - auch für Gesellschaften mit ausländischer Rechtsform gelten, die in Deutschland ihren Verwaltungssitz haben. ${ }^{162}$

Aus Gründen der Rechtssicherheit wird vorgeschlagen, die relevanten ausländischen Rechtsformen enumerativ in $\S 1$ MitbestG aufzunehmen. ${ }^{63}$ Für monistisch strukturierte Auslandsgesellschaften könnte sich die Mitbestimmung auf die nicht geschäftsführenden Verwaltungsratsmitglieder beschränken, um eine Ausweitung der materiellen Arbeitnehmerrechte zu verhindern. ${ }^{64}$ Insofern kann auf die Modelle zur Integration der Mitbestimmung bei der monistischen SE verwiesen werden. Beispielhaft kann insofern die monistische Puma SE betrachtet werden. ${ }^{65}$ Sie wird durch einen Verwaltungsrat geleitet, wobei die Tagesgeschäfte nicht von diesem, sondern von den geschäftsführenden Direktoren nach Vorgaben und Weisungen des Verwaltungsrats getätigt werden. ${ }^{66}$ Die Arbeitnehmermitbestimmung (bei Puma nach dem Drittelbeteiligungsgesetz) ist im Verwaltungsrat verortet; dieser setzt sich aus sechs Anteilseigner-Mitgliedern und drei Arbeitnehmervertretern zusammen ${ }_{i}{ }^{67}$ letztere werden auf Grund eines bindenden Arbeitnehmervertretervorschlags gewählt. ${ }^{68}$ Die unternehmerische Mitbestimmung der Arbeitnehmer im Verwaltungsrat der SE wird in einer Arbeitnehmerbeteiligungsvereinbarung näher geregelt. ${ }^{69}$

61 BT-Drucks. 17/2122, S. 2.

62 Antrag „Unternehmensmitbestimmung lückenlos garantieren“, BT-Drucks. 17/1413, S. 1.

63 Sick, GmbHR 2011, 1196, 1198.

64 Sick, GmbHR 2011, 1196, 1198; Weller in MünchKomm. GmbHG, 2010, Int. GesR, Einl., Rz. 480.

65 Sick, GmbHR 2011, 1196, 1198.

$66 \mathrm{Vgl}$. § 6.3 Puma SE-Satzung sowie $\S \S 1.1,3.2$ Geschäftsordnung Verwaltungsrat PUMA SE (Stand 2011). Vorsitzender der geschäftsführenden Direktoren ist der Chief Executive Officer, der gleichzeitig Verwaltungsratsmitglied sein kann, § 6.1 GO Verwaltungsrat; $\S \S 2.2,5.1$ und 5.4 GO für die Geschäftsführenden Direktoren.

67 Umwandlungsbericht des Vorstands der PUMA AG, 2011, S. 25.

68 S. §§ 7.1, 7.3 SE-Satzung; 1.5 GO Verwaltungsrat; PUMA Einladungsbekanntmachung 2011, Anlage I, verfügbar unter www.about.puma.com (abgerufen am 5.12.2011).

69 § 21 SEBG; Umwandlungsbericht des Vorstands der PUMA AG, S. 31, verfügbar unter www.about.puma.com. Im Falle des Nicht-Abschlusses einer Mitbestimmungsvereinbarung greift eine Arbeitnehmerbeteiligung nach den Auffangregelungen des SEBG ein, $§ \S 22 \mathrm{ff}$., $34 \mathrm{ff}$. SEBG. 
Ungeachtet dessen, wie man rechtspolitisch zur Mitbestimmung steht, drängt sich jedenfalls die rechtskonstruktive Frage auf, ob sich die Mitbestimmung über ein solches Erstreckungsgesetz systemkohärent auf Auslandsgesellschaften ausdehnen ließe. Dieser Frage soll im Folgenden nachgegangen werden.

\section{Unternehmensmitbestimmung für Auslandsgesellschaften aus Sicht des IPR}

Bei Sachverhalten mit Auslandsbezug entscheidet gemäß Art. 3 EGBGB das IPR als rechtliche Metaordnung, welche Rechtsordnung auf einen bestimmten Sachverhaltskomplex Anwendung findet. Hierfür bedient sich das IPR verschiedener Kollisionsregeln, wobei man zwischen "regulären" Anwendungsbefehlen auf Grundlage materiellrechtlich neutraler (allseitiger) Kollisionsregeln im Sinne Savignys ${ }^{70}$ und „irregulären" Anwendungsbefehlen auf Grundlage einseitiger Eingriffsnormen differenzieren kann.

\section{Unternehmensmitbestimmung via Gesellschaftsstatut}

Die Unternehmensmitbestimmung ist nicht arbeitsrechtlich, sondern gesellschaftsrechtlich zu qualifizieren, ${ }^{71}$ so dass die (allseitige) gesellschaftsrechtliche Kollisionsregel einschlägig ist. Für die gesellschaftsrechtliche Qualifikation spricht der Zweck des geltenden Mitbestimmungsrechts: Es zielt auf die gleichberechtigte Teilhabe der Arbeitnehmer an den unternehmerischen Entscheidungen und ihren Abläufen und somit auf die Chance, Beschlussfassungen auf Ebene der Unternehmensführung zu beeinflussen. ${ }^{72}$ Folgerichtig ist die Unternehmensmitbestimmung anders als die betriebliche Mitbestimmung in einem Organ der Gesellschaftsverfassung, dem Aufsichtsrat, verankert.

Auf Grundlage der gesellschaftsrechtlichen Kollisionsregel ist ein Anwendungsbefehl für das MitbestG 1976 in Bezug auf Auslandsgesellschaften zu verneinen, soweit es um Auslandsgesellschaften geht, deren Gesellschaftstatut nach der Gründungstheorie ermittelt wird; denn in einem solchen Fall findet deutsches Gesellschaftsrecht (und damit auch das MitbestG 1976 als Teil des inländischen Gesellschaftsrechts) gerade keine Anwendung. ${ }^{73}$ Auf gesellschaftskollisionsrechtlicher Grundlage kann das MitbestG folglich nicht auf EU- und US-Gesellschaften mit inländischem Verwaltungssitz herangezogen werden. ${ }^{74}$

70 Zur Struktur klassischer Kollisionsregeln i.S. Savignys Weller, IPRax 2011, 429, $430 \mathrm{ff}$.

71 Hübner, Kollisionsrechtliche Behandlung von Gesellschaften aus „nicht-privilegierten" Drittstaaten, 2010, S. 235; Kindler in MünchKomm. BGB, 5. Aufl. 2010, Int. GesR, Rz. 565; Eberspächer, ZIP 2008, 1951.

72 Vgl. die Gesetzesbegründung zum MitbestG 1976, BT-Drucks. VII/2172, S. 16; hierzu Hommelhoff, ZGR 2010, 48, 52.

73 Hübner, Kollisionsrechtliche Behandlung von Gesellschaften aus „nicht-privilegierten" Drittstaaten, 2010, S. 241 f.; Eberspächer, ZIP 2008, 1951.

74 Henssler in GS Heinze, 2005, S. 333 ff.; Eberspächer, ZIP 2008, 1951, 1958; Ebke, IZ 2003, 927, $930 \mathrm{f}$. 


\section{Unternehmensmitbestimmung via Eingriffsstatut}

Allerdings kommt eine Durchsetzung der inländischen Unternehmensmitbestimmung als Eingriffsnorm, d.h. via Sonderanknüpfung aufgrund international zwingenden Charakters in Betracht. ${ }^{75}$ Man kann von einem irregulären Anwendungsbefehl sprechen, weil sich auf diese Weise ein inländisches Rechtsinstitut einseitig gegen ein an sich regulär zur Anwendung berufenes ausländisches Sachstatut (z.B. gegen das englische Gesellschaftsstatut) durchsetzt; es kommt mithin zu einer punktuellen Überlagerung des regulär anwendbaren ausländischen Statuts durch ein inländisches Institut.

Das methodische Instrument zur irregulären Durchsetzung inländischer Institute ist entgegen verbreiteter Auffassung indes nicht der ordre public, weil diesem im deutschen Recht nur eine negative Abwehrfunktion, nicht jedoch eine positive Durchsetzungsfunktion zukommt (vgl. Art. 6 EGBGB). Einschlägig zur Durchsetzung inländischer Normen und Rechtsfiguren gegen ein an sich zur Anwendung berufenes ausländisches Sachstatut ist vielmehr die Lehre von der Sonderanknüpfung international zwingender Bestimmungen.

\section{Lehre von der Sonderanknüpfung international zwingender Normen}

\section{a) Wilhelm Wengler}

Die Lehre von der Sonderanknüpfung international zwingender Normen geht auf Wilhelm Wengler zurück. ${ }^{76}$ Hiernach beherrscht eine Rechtsordnung ein Rechtsverhältnis nur insoweit, als nicht einzelne Fragen gesondert anzuknüpfen und einem anderen Recht zu unterstellen sind. ${ }^{77}$ Letzteres ist insbesondere bei Sachnormen der lex fori der Fall, die in dem Umfang zur Anwendung gelangen, „wie es dem politischen Interesse des Heimatstaates des Richters entspricht" ${ }^{\prime 78}$. Sofern durch Interpretation nach Sinn und Zweck einer inländischen Bestimmung ihr universaler Anwendungswille ermittelt werden kann, ist sie durch Sonderanknüpfung auf das Rechtsverhältnis anzuwenden, und zwar „ganz gleich ob auf das Rechtsverhältnis auch im übrigen - sei es kraft Parteiautonomie oder kraft der subsidiär anwendbaren staatlichen Kollisionsnormen - die lex fori (...) anwendbar ist oder nicht ${ }^{\prime \prime 79}$. Zwingende Normen schalten folglich den gewöhnlichen Mechanismus der Verweisung aus und sind unabhängig vom internationalprivatrechtlich angeknüpften Recht anzuwenden. ${ }^{80}$

75 Hübner, Kollisionsrechtliche Behandlung von Gesellschaften aus „nicht-privilegierten" Drittstaaten, 2010, S. $241 \mathrm{f}$.

76 Wengler, ZVglRWiss 54 (1941), $168 \mathrm{ff}$.

77 Wengler, ZVglRWiss 54 (1941), 168, 211.

78 Wengler, ZÖR 23 (1944), 473, 486.

79 Wengler, ZVglRWiss $54(1941), 168,178$.

80 Sonnenberger, IPRax 2003, 104, 105. 


\section{b) Abgrenzung zum ordre public}

Auch wenn Wengler auf eine gewisse "innere Verwandtschaft" zwischen ordre public und „international zwingenden Bestimmungen“ im Blick auf die jeweils dahinterstehenden politischen Interessen des Forumstaates hinweist, ${ }^{81}$ unterstreicht er doch zugleich die methodischen Unterschiede, die letztlich dazu geführt haben, dass die Lehre von der generellen positiven Funktion des ordre public von der Lehre von der Sonderanknüpfung zwingender Normen verdrängt worden ist: ${ }^{82}$ Beim ordre public wird das materielle Ergebnis der Anwendung ausländischen Rechts mit dem Inhalt der entsprechenden deutschen Sachnorm verglichen, wobei deren Inhalt Bestandteil der wesentlichen Grundsätze des deutschen Rechts (Art. 6 EGBGB) sein muss. ${ }^{83}$ Der ordre public verfolgt damit eine Außenperspektive, indem er auf die Akzeptanz des fremden Rechtsinhalts blickt. ${ }^{84}$ Dagegen kommt es bei der Sonderanknüpfung von inländischen Sachnormen überhaupt nicht auf den Inhalt des ausländischen Rechts an. Auch der Inhalt der deutschen Sachnorm hat an sich nur geringe Relevanz. ${ }^{85}$ Vielmehr ist allein die Bestimmung und Abgrenzung des örtlichen Geltungsbereichs der inländischen Sachnorm von Bedeutung. ${ }^{86}$ Indem nach der Reichweite ihres sachlichen und räumlichen Anwendungs- bzw. Geltungswillens gefragt wird, ${ }^{87}$ hat die Lehre von der Sonderanknüpfung zwingender Normen eine - wie Magnus ${ }^{88}$ es treffend ausdrückt - „Innenperspektive im Auge und erweitert den Geltungsanspruch des internen Rechts". Anders gewendet: Da der Inhalt der Sachnorm für ihre Qualifikation als zwingende Norm nicht im Mittelpunkt steht, brauchen zwingende Normen im Unterschied $z \mathbf{u}$ ordre public-relevanten Rechtssätzen keine wesentlichen Grundsätze der deutschen Rechtsordnung zu schützen, haben also nicht per se ordre public-Charakter. ${ }^{89}$

\section{Sonderanknüpfung im Gesellschaftsrecht}

Der Begriff der international zwingenden Bestimmungen hat im europäischen Schuldvertragsrecht in Art. 7 EVÜ sowie nunmehr in Art. 9 Rom I-VO eine Kodifikation erfahren. Art. 1 Abs. 2 (f) Rom I-VO stellt jedoch klar, dass Art. 9 Rom I-VO nicht für gesellschaftsrechtliche Fragen gilt, so dass eine Sonderanknüpfung der Unternehmensmitbestimmung nicht unmittelbar auf Art.9 Rom I-VO gestützt werden kann. Allerdings ist anerkannt, dass die zwingenden Bestimmungen kein Sonderthema des Internationalen Schuldvertragsrechts

81 Wengler, ZÖR 23 (1944), 473, 486.

82 Jayme, Methoden der Konkretisierung des ordre public im Internationalen Privatrecht, 1989, S. 28

83 Wengler, ZÖR 23 (1944), 473, $486 \mathrm{f}$.

84 Magnus in Staudinger, Neubearb. 2011, Rom I-VO, Art. 9, Rz. 31.

85 Sonnenberger, IPRax 2003, 104, 109.

86 Wengler, ZVglRWiss 54 (1941), 168, 173 ff., 178; ders., ZÖR 23 (1944), 473, 486 f.

87 Tayme, IPRax 2001, 190.

88 Magnus in Staudinger, Neubearb. 2011, Rom I-VO, Art. 9, Rz. 31.

89 Martiny in MünchKomm. BGB, 5. Aufl. 2010, VO (EG) 593/2008, Art. 9, Rz. 111. 
sind, sondern in allen Bereichen des IPR vorkommen können. ${ }^{90}$ Art. 9 Rom IVO ist vielmehr nur ein punktuell-kodifizierter Ausschnitt des im IPR allgemein anerkannten Grundsatzes, dass die Kollisionsnormen nicht die Anwendung jener Bestimmungen der lex fori berühren, die den Sachverhalt ohne Rücksicht auf das für das Rechtsverhältnis maßgebende Recht zwingend regeln. ${ }^{91}$ Bestätigt wird dies durch Art. 16 Rom II-VO, welcher für den Bereich der außervertraglichen Schuldverhältnisse gleichfalls von der Existenz von Eingriffsnormen ausgeht. Eine Sonderanknüpfung der Unternehmensmitbestimmung kann daher über die allgemein anerkannte Lehre von der Sonderanknüpfung international zwingender Normen erfolgen, sofern deren Voraussetzungen gegeben sind.

\section{Allgemeine Voraussetzungen einer Sonderanknüpfung}

Ein Gesetzgeber kann eine Sonderanknüpfung - vorbehaltlich ihrer Europarechtskonformität (s. unter E.) - ausdrücklich anordnen, wie es etwa in $\S 130$ Abs. 2 GWB $^{92}$ geschehen ist. Kollisionsrechtlich systemkohärent ist eine solche Sonderanknüpfung, wenn sie zwei Anforderungen genügt: ${ }^{93}$

(1) Zum einen sollte sie sich auf Sachverhalte beschränken, die einen hinreichenden Bezug zum Inland aufweisen. ${ }^{94}$ Dies würde für ein Mitbestimmungs-Erstreckungsgesetz bedeuten, dass sich sein Anwendungsbereich auf Auslandsgesellschaften beschränkt, die ihren organisatorischen Mittelpunkt in Deutschland haben. ${ }^{95}$ Bei Auslandsgesellschaften wie etwa Air Berlin, deren effektiver Verwaltungssitz sowie deren Tätigkeitsschwerpunkt im Inland liegt, wäre eine solcher enger Inlandsbezug zu bejahen.

(2) Zum anderen muss die durchzusetzende Norm als international zwingend zu qualifizieren sein. ${ }^{96}$ Für international zwingende Bestimmungen wird auch heute noch ungeachtet einiger Kritik ${ }^{97}$ der von Neuhaus ${ }^{98}$ geprägte Terminus der Eingriffsnormen synonym verwandt (so z. B. Art. 16 Rom II-VO), wobei anerkannt ist, dass es hierbei um Normen geht, die im öffentlichen Interesse Eingriffe in private Rechtsverhältnisse ermöglichen. ${ }^{99}$ Nach herrschender Auf-

90 Sonnenberger, IPRax 2003, 104.

91 Tayme, Methoden der Konkretisierung des ordre public im Internationalen Privatrecht, 1989, S. 29.

$92 \S 130$ Abs. 2 GWB bestimmt: "Dieses Gesetz findet Anwendung auf alle Wettbewerbsbeschränkungen, die sich im Geltungsbereich dieses Gesetzes auswirken, auch wenn sie außerhalb des Geltungsbereichs dieses Gesetzes veranlasst werden."

93 Hübner, Kollisionsrechtliche Behandlung von Gesellschaften aus „nicht-privilegierten" Drittstaaten, 2010, S. 242; ausführliche Begründung bei Weller in MünchKomm. GmbHG, 2010, Int. GesR, Einl., Rz. 450-458.

94 Magnus in Staudinger, Neubearb. 2011, Rom I-VO, Art. 9, Rz. 46, 81 ff.

95 Vgl. auch Sick, GmbHR 2011, 1196, 1197.

96 Martiny in MünchKomm. BGB, 5. Aufl. 2010, VO (EG) 593/2008, Art. 9, Rz. 107.

97 Tayme, IPRax 2001, 190, 191: „Es gilt Abschied zu nehmen von dem voreuropäischen Begriff der Eingriffsnormen". Für die Beibehaltung des Begriffs „Eingriffsrecht" dagegen Sonnenberger, IPRax 2003, 104, $105 \mathrm{f}$.

98 Neuhaus, Grundbegriffe des Internationalen Privatrechts, 2. Aufl. 1976, S. 33 ff.

99 Sonnenberger in MünchKomm. BGB, 5. Aufl. 2010, EGBGB, Einl. IPR, Rz. 35 f. 
fassung ${ }^{100}$ ist für den internationalen Anwendungswillen einer sonderanzuknüpfenden Sachnorm maßgeblich, ob sich ihr Zweck nicht im Ausgleich widerstreitender Interessen der Vertragsparteien und daher Individualbelangen erschöpft, sondern zumindest auch auf Gemeinwohlinteressen gerichtet ist. ${ }^{101}$ Mithin ist die Gemeinwohlorientierung der in Frage stehenden Normen entscheidend, welche bei Vorschriften, die ausschließlich im Dienste des privaten Rechtsverkehrs stehen (sog. Parteischutzvorschriften), nicht gegeben ist. ${ }^{102}$ Eine international zwingende Norm muss neben der Regelung von Privatbeziehungen darüber hinaus auch Interessen verfolgen, die über den Einzelnen, über die Privatperson hinausgehen. ${ }^{103}$ Zum Kernbereich der international zwingenden Bestimmungen zählen insbesondere die auch in Art. 9 Abs. 1 Rom I-VO genannten wirtschafts- und sozialpolitischen Vorschriften. ${ }^{104}$

\section{Sonderanknüpfung des MitbestG 1976}

Die Regeln zur unternehmerischen Mitbestimmung können vor diesem Hintergrund als sog. international zwingende Bestimmungen des deutschen Rechts qualifiziert werden. ${ }^{105}$ Der deutsche Gesetzgeber hat den international zwingenden Charakter des MitbestG 1976 zwar nicht explizit angeordnet, allerdings ergibt er sich aus seinem Normzweck. Das MitbestG 1976 geht über einen bloßen Interessenausgleich zwischen Privaten deutlich hinaus; es zielt maßgeblich auf die Verwirklichung sozialpolitischer und gesellschaftlicher Ziele. Es bezwecke nämlich - so das Bundesverfassungsgericht ${ }^{106}$-, die Fremdbestimmung von Arbeitnehmern in größeren Unternehmen zu mildern und die ökonomische Legitimation durch eine soziale Komponente zu ergänzen; verwirklicht werde dabei nicht nur ein (privates) "Gruppeninteresse", vielmehr habe die durch die Mitbestimmung angestrebte Kooperation und Integration „allgemeine gesellschaftspolitische Bedeutung "107. Die Mitbestimmung sei zur politischen Sicherung der Marktwirtschaft geeignet und diene dem Wohle der Allgemeinheit. ${ }^{108}$ Auch kann sie als Gewährleistungsinstrument für eine soziale Unternehmenspolitik als „Bestandteil der deutschen Sozialordnung“ eingeordnet werden. ${ }^{109}$ Ausweislich des Gesetzgeberwillens gehöre die Mitbestimmung „zur Substanz des Demokratisierungsprozesses“, sei Voraussetzung für Reformen des freiheitlichen Sozialstaats ${ }^{110}$ und schütze damit öffent-

100 BGH v. 27.2.2003 - VII ZR 169/02, IPRax 2003, 449, 451; Sonnenberger, IPRax 2003, $104,107 \mathrm{ff}$.

101 BAGE $63,17,32 ;$ BAGE $71,297,317$.

102 Sonnenberger, IPRax 2003, 104, 107 ff.; Bälz, NIW 2003, 1559, 1562.

103 BGH v. 27.2.2003 - VII ZR 169/02, IPRax 2003, 449, 451; Sonnenberger, IPRax 2003, $104,107 \mathrm{ff}$.

104 Sonnenberger, IPRax 2003, 104, $107 \mathrm{ff}$.

105 Näher Kindler in MünchKomm. BGB, 5. Aufl. 2010, Int. GesR, Rz. 572.

106 BVerfG v. 1.3.1979, BVerfGE 50, 290 - „Mitbestimmung“.

107 BVerfGE 50, 290, $350 \mathrm{f}$.

108 BVerfGE 50, 290, 351.

109 Henssler in GS Heinze, 2005, S. 333, 347.

110 BT-Drucks. VII/2172, S. 17. 
liche Interessen. ${ }^{111}$ Diese bestünden z. B. in der Demokratisierung wirtschaftlicher Entscheidungsprozesse, der Gewährleistung der Menschenwürde der Arbeitnehmer, die nicht zum bloßen Vollzugsobjekt fremder Entscheidungen degradiert werden dürften, der Schaffung sozialen Friedens in der Gesellschaft durch Überwindung einer drohenden „Klassenkämpfermentalität" und der Kontrolle wirtschaftlicher Macht als historischem Anliegen des Arbeitsrechts. ${ }^{112}$ Nach alledem ließe sich ein Mitbestimmungs-Erstreckungsgesetz aus Sicht des IPR als Eingriffsnorm systemkohärent legitimieren.

\section{E. Unternehmensmitbestimmung für Auslandsgesellschaften aus Sicht des Europarechts}

\section{Europarechtskonformität der Sonderanknüpfung?}

In europarechtlicher Hinsicht müssen die auf Scheinauslandsgesellschaften anzuwendenden inländischen Regelungen - ungeachtet des jeweiligen kollisionsrechtlichen Anwendungsbefehls (d.h. unabhängig davon, ob sie „regulär" oder „irregulär“ im Wege der Sonderanknüpfung Geltung beanspruchen) - im Einklang mit den Grundfreiheiten, insbesondere mit der Niederlassungsfrei-

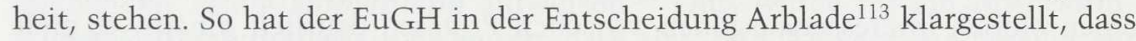
selbst Institute, die aus Perspektive der lex fori zum ordre public oder zu den international zwingenden Bestimmungen gerechnet werden, gleichwohl mit den Grundfreiheiten harmonieren müssen. ${ }^{114} \mathrm{Da}$ ein Mitbestimmungs-Erstreckungsgesetz einen grundfreiheitsbeschränkenden Charakter hat (dazu unter II.), müsste es dem vierstufigen Rechtfertigungstest der sog. Cassis de Dijon- bzw. der Gebhard-Formel ${ }^{115}$ standhalten, um europarechtskonform zu sein /dazu unter III). ${ }^{116}$ Im Einzelnen:

111 Rehberg in Eidenmüller, Ausländische Kapitalgesellschaften im deutschen Recht, $\S 6 \mathrm{Rz} .40 \mathrm{ff} ., 113$.

112 Rehberg in Eidenmüller, Ausländische Kapitalgesellschaften im deutschen Recht, $\S 6 \mathrm{Rz} .42 \mathrm{ff}$.

113 EuGH v. 23.11.1999 - C-369/96 und C-376/96, Slg. 1999, I-8453 - Arblade.

114 Jayme/Kohler, IPRax 2000, 454, 455; Behrens, IPRax 2003, 193, 206.

115 EuGH v. 30.11.1995 - Rs. C-55/94, Slg. 1995, I-4165 - Gebhard, Tz. 37: „Aus der Rechtsprechung des Gerichtshofes ergibt sich jedoch, dass nationale Maßnahmen, die die Ausübung der durch den Vertrag garantierten grundlegenden Freiheiten behindern oder weniger attraktiv machen können, vier Voraussetzungen erfüllen müssen: [1.] Sie müssen in nichtdiskriminierender Weise angewandt werden, [2.] sie müssen aus zwingenden Gründen des Allgemeininteresses gerechtfertigt sein, [3.] sie müssen geeignet sein, die Verwirklichung des mit ihnen verfolgten Zieles zu gewährleisten, [4.] und sie dürfen nicht über das hinausgehen, was zur Erreichung dieses Zieles erforderlich ist."

116 Hübner, Kollisionsrechtliche Behandlung von Gesellschaften aus "nicht-privilegierten" Drittstaaten, 2010, S. 242 ff.; Zimmer, NJW 2003, 3585, $3591 \mathrm{f}$. 


\section{Mitbestimmungs-Erstreckungsgesetz als Beschränkung der Niederlassungsfreiheit}

Seit der Gebhard-Entscheidung des EuGH gelten als Beschränkung aller vier Grundfreiheiten alle „nationale[n] Maßnahmen, die die Ausübung der durch den Vertrag garantierten grundlegenden Freiheiten behindern oder weniger attraktiv machen können."117 Diese Formulierung knüpft an die für die Warenverkehrsfreiheit entwickelte Dassonville-Formel an, ${ }^{118}$ nach der "jede Handelsregelung der Mitgliedstaaten, die geeignet ist, den innergemeinschaftlichen Handel unmittelbar oder mittelbar, tatsächlich oder potenziell zu behindern, als Maßnahme gleicher Wirkung wie eine mengenmäßige Beschränkung anzusehen " ist. ${ }^{119}$ In der Centros-Entscheidung ${ }^{120}$ erkannte der EuGH die Uferlosigkeit seiner Beschränkungsformel ${ }^{121}$ und deutete - wohl in Analogie zur Keck-Rechtsprechung ${ }^{122}$ zur Warenverkehrsfreiheit - eine Unterscheidung zwischen Regeln „über die Errichtung von Gesellschaften”, die von der Literatur als am Beschränkungsverbot zu messende Marktzugangsregelungen aufgefasst werden, und Tätigkeitsausübungsregelungen an, die entweder nicht in den Schutzbereich der Niederlassungsfreiheit fallen oder jedenfalls einer leichteren Rechtfertigung zugänglich sein sollen. ${ }^{123}$

Vor diesem Hintergrund stellt die sonderanknüpfungsbasierte Erstreckung der Unternehmensmitbestimmung auf EU-Auslandsgesellschaften mit Verwaltungssitz in Deutschland eine Beschränkung der Niederlassungsfreiheit dar. ${ }^{124}$ Denn durch das Aufzwingen eines vom Gründungsrecht abweichenden Mitbestimmungsmodells werden der Gesellschaft zusätzliche Pflichten auferlegt, die sie nach ihrem Gründungsrecht nicht zu beachten hat. ${ }^{125}$ Angesichts dessen, dass sie die rechtliche Verfasstheit und Organisationsstruktur einer Gesellschaft maßgeblich modifiziert, handelt es sich nicht um eine bloße Tätigkeitsausübungsregel, sondern um eine Marktzutrittsregel, die umfänglich dem Beschränkungsverbot unterfällt. ${ }^{126}$ Eine Erstreckung der unternehmerischen

117 EuGH v. 30.11 .1995 - Rs. C-55/94, Slg. 1995, I-4165 - Gebhard; s. auch jüngst EuGH v. 29.3.2011 - Rs. C-565/08, NJW 2011, 1575: Maßnahmen, die die Ausübung „verbieten, behindern oder weniger attraktiv machen"; Weller, Europäische Rechtformwahlfreiheit und Gesellschafterhaftung, 2004, S. $34 \mathrm{f}$.

118 Weller, Europäische Rechtformwahlfreiheit und Gesellschafterhaftung, 2004, S. 35.

119 EuGH v. 11.7.1974 - Rs. 8-74, Slg. 1974, 837, 852 - Dassonville.

120 EuGH v. 9.3.1999 - Rs. C-212/97, Slg. 1999, I-1459 - Centros.

121 Weller, Europäische Rechtsformwahlfreiheit und Gesellschafterhaftung, 2004, S. 35.

122 EuGH v. 24.11.1993 - Rs. C-267/91 und C-268/91, Slg. 1993, I-6097 - Keck und Mithouard.

123 Kainer, Unternehmensübernahmen im Binnenmarktrecht, 2004, S. 118 ff.; Weller, Europäische Rechtsformwahlfreiheit und Gesellschafterhaftung, 2004, S. 34 ff.

124 Hübner, Kollisionsrechtliche Behandlung von Gesellschaften aus „nicht-privilegierten" Drittstaaten, 2010, S. 243; Braun, Die Sicherung der Unternehmensmitbestimmung im Lichte des europäischen Rechts, 2005, S. 175; Eberspächer, ZIP 2008, 1951, 1953; Teichmann, ZIP 2009, 1787, 1788; Veit/Wichert, AG 2004, 14, 18.

125 Thüsing, ZIP 2004, 381, 385.

126 Rehberg in Eidenmüller, Ausländische Kapitalgesellschaften im deutschen Recht, $\S 6$ Rz. 22; Braun, Die Sicherung der Unternehmensmitbestimmung im Lichte des europäischen Rechts, 2005, S. 175; Forsthoff, DB 2002, 2471, 2477. 
Mitbestimmung auf EU-Auslandsgesellschaften wäre nach alledem nur dann unionsrechtskonform, wenn sie sich rechtfertigen ließe.

\section{Rechtfertigung der Anwendung der Unternehmensmitbestimmung auf Auslandsgesellschaften}

\section{Zwingender Grund des Allgemeininteresses}

Der EuGH hat in Überseering in einem obiter dictum geäußert, der Schutz der Arbeitnehmer stelle einen zwingenden Grund des Allgemeininteresses im Sinne der Cassis-/Gebhard-Formel dar, der eine Beschränkung der Niederlassungsfreiheit einer ausländischen Gesellschaft rechtfertigen könne. ${ }^{127} \mathrm{Die}$ Qualifikation der Unternehmensmitbestimmung als zwingender Grund wird durch die Richtlinie 2005/56/EG über die Verschmelzung von Kapitalgesellschaften erhärtet, respektiert diese doch die verschiedenen Mitbestimmungsregime der Mitgliedstaaten. Insbesondere soll gemäß Art. 16 der Richtlinie auch bei grenzüberschreitenden Verschmelzungen die bei der Societas Europaea (SE) vorgesehene Regelung zur Arbeitnehmermitbestimmung, bestehend aus Verhandlungsverfahren und subsidiärer Auffanglösung in Gestalt eines mitbestimmungsrechtlichen Bestandsschutzes, zur Anwendung kommen. ${ }^{128}$ Ziel dieser Bestimmung ist es gerade zu verhindern, dass die unternehmerische Arbeitnehmermitbestimmung umgangen wird, indem die aus der Verschmelzung hervorgehende Gesellschaft in einem Mitgliedstaat errichtet wird, der kein Mitbestimmungsrecht kennt. ${ }^{129}$

Abgesehen davon, dass die Mitbestimmung hiernach durchaus ein schon auf Unionsebene anerkanntes Gemeinwohlinteresse ist, würde es m.E. für die Cassis-Rechtfertigung auch ausreichen, wenn sie lediglich aus Sicht des deutschen Rechts als zwingender Grund des Allgemeininteresses einzustufen wäre. Demgegenüber überzeugt es nicht, wenn gewichtige Stimmen ${ }^{130}$ dafür plädieren, als Rechtfertigungsgrund kämen nur europäische im Sinne von europaweit, d.h. in allen Mitgliedstaaten anerkannte Allgemeinwohlinteressen in Frage (was bei der paritätischen Mitbestimmung nicht der Fall ist), nicht jedoch Interessen, die allein aus inländischer Sicht Gemeinwohlbedeutung haben. Vielmehr zeigt das Europäische Kollisionsrecht bei den Figuren der Sonderanknüpfung und des ordre public (z.B. Art. 9, 21 Rom I-VO, Art. 34 Nr. 1 EuGVO|, dass die Einnahme einer nationalen Perspektive bei der Bestimmung von Gemeinwohlinteressen sehr wohl europarechtskonform ist.

127 EuGH v. 5.11.2002 - Rs. C-208/00, ZIP 2002, 2037, Tz. 89, 92 - Überseering.

128 Zur Mitbestimmung in der SE Reichert/Brandes, ZGR 2003, 767; Heinze, ZGR 2003, 66, 80 ff.; Gruber/Weller, NZG 2003, 297.

129 In Deutschland wurde Art. 16 der Richtlinie in dem „Gesetz über die Mitbestimmung der Arbeitnehmer bei einer grenzüberschreitenden Verschmelzung (MgVG) umgesetzt, MgVG v. 21.12.2006, BGBl. I 2006, 3332.

130 So Hübner, Kollisionsrechtliche Behandlung von Gesellschaften aus ,nicht-privilegierten" Drittstaaten, 2010, S. 244; Ebke, JZ 2003, 927, 931. 


\section{Geeignetheit}

Ein Mitbestimmungs-Erstreckungsgesetz wäre im Sinne der Cassis-/GebhardFormel auch geeignet, dem Schutz der Arbeitnehmer zu dienen, da es den Geltungsbereich des MitbestG 1976 auf Auslandsgesellschaften mit hinreichendem Inlandsbezug erstrecken würde. ${ }^{131}$ Ein Blick auf die Gesetzgebungskompetenz des Art. 153 Abs. 1 lit. f AEUV $^{132}$ bestätigt, dass das Unionsrecht die Mitbestimmung als grundsätzlich geeignet zur Förderung des sozialen Schutzes der Arbeitnehmer ansieht, da andernfalls die Aufnahme der "Mitbestimmung" in Art. 153 AEUV widersprüchlich gewesen wäre. ${ }^{133}$

\section{Erforderlichkeit}

Allerdings dürfte die die Niederlassungsfreiheit beschränkende nationale Maßnahme nach der Cassis-/Gebhard-Formel nicht über das hinausgehen, was zur Erreichung des verfolgten Zieles erforderlich ist. ${ }^{134}$ Dieser sog. SchrankenSchranke kann ein Mitbestimmungs-Erstreckungsgesetz unter bestimmten, sogleich auszuführenden Voraussetzungen, gerecht werden.

\section{a) Betriebliche Mitbestimmung als Alternative?}

Die Erforderlichkeit entfiele, wenn es ein milderes Mittel hinsichtlich der Interessenwahrung der Arbeitnehmer gäbe. Ein solches milderes Mittel sehen manche in der Erstreckung der betrieblichen Mitbestimmung auf Auslandsgesellschaften. ${ }^{135}$ Jedoch ist die betriebliche Mitbestimmung wegen der unterschiedlichen Funktionen der betrieblichen und unternehmerischen Mitbestimmung kein funktional adäquater Ersatz. ${ }^{136}$ Durch die betriebliche Mitbestimmung erlangen die Arbeitnehmer insbesondere keine Teilhabe an der strategischen "Steuerung des Unternehmens im Ganzen",137 worauf die unternehmerische Mitbestimmung jedoch gerade abzielt.

\section{b) Notwendigkeit einer Subsidiaritätsklausel?}

In der Literatur wird angeführt, eine Mitbestimmungserstreckung sei nicht erforderlich, soweit das Recht des Gründungsstaats ein vergleichbares Arbeit-

131 Ähnlich Thüsing, ZIP 2004, 381, 387; Eberspächer, ZIP 2008, 1951, 1954; Henssler in GS Heinze, 2005, S. 333, 353.

132 „Zur Verwirklichung der Ziele des Artikels 151 unterstützt und ergänzt die Union die Tätigkeit der Mitgliedstaaten auf folgenden Gebieten: ... f) Vertretung und kollektive Wahrnehmung der Arbeitnehmer- und Arbeitgeberinteressen einschließlich der Mitbestimmung".

133 Vgl. Thüsing, ZIP 2004, 381, 387.

134 EuGH v. 30.11 .1995 - Rs. C-55/94, Slg. 1995, I-4165 - Gebhard.

135 Eidenmüller, ZIP 2002, 2233, 2242; Seibt in Henssler/Willemsen/Kalb, Arbeitsrecht: Kommentar, 2010, §1 MitbestG, Rz. 8; Forsthoff, DB 2002, 2471, 2477; Müller-Bonnani, GmbHR 2003, 1235, 1238.

136 Weiss/Seifert, ZGR 2009, 542, 564 ff.; Thüsing, ZIP 2004, 381, 387; Bayer, AG 2004, $534,538$.

137 Weiss/Seifert, ZGR 2009, 542, 565. 
nehmerschutzniveau durch Mitbestimmung vorsieht. ${ }^{138}$ Die Beibehaltung des ausländischen Mitbestimmungsrechts wäre milder, weil Eingriffe in die Organisationsverfassung der Gesellschaft unterblieben. ${ }^{139}$ Folgt man dem, müsste ein deutsches Erstreckungsgesetz eine Subsidiaritätsklausel enthalten, derzufolge eine vergleichbare Mitbestimmungsregelung des Gründungsstaats Vorrang hat. ${ }^{140}$ Diese Ansicht widerspricht zwar der - auch vom Unionsrecht anerkannten (vgl. Art. 9 Rom I-VO) - kollisionsrechtlichen Dogmatik der Sonderanknüpfung, wonach es auf den Inhalt des ausländischen Rechts gerade nicht ankommt; allerdings wird das autonome deutsche sowie das unions-sekundärrechtliche IPR für innereuropäische Sachverhalte durch die primärrechtliche Grundfreiheitendogmatik überlagert und insofern durch das Erforderlichkeitskriterium der Cassis-/Gebhard-Formel modifiziert. Daher verfängt das Petitum nach einer Subsidiaritätsklausel.

\section{c) Unternehmensmitbestimmung in monistischen Auslandsgesellschaften}

Bedenken werden schließlich insoweit geäußert, als monistisch strukturierten Gesellschaften ein zusätzliches Aufsichtsorgan auferlegt werden solli ${ }^{141}$ die „Einpflanzung" eines Aufsichtsrats berge die Gefahr, dass das austarierte Interessensystem aus dem Gleichgewicht gerate. ${ }^{142}$

Diese Bedenken überzeugen nicht. Ein Verstoß gegen das im Erforderlichkeitskriterium der Gebhard-Formel ${ }^{143}$ enthaltene "Übermaßverbot" liegt selbst bei monistisch verfassten EU-Auslandsgesellschaften nicht vor, sofern man sich an dem aus dem SE-Statut bekannten und in der Internationalen Verschmelzungsrichtlinie aufgegriffenen Modell der Verhandlungslösung orientiert. ${ }^{144}$ Dieses ermöglicht es, die Arbeitnehmermitbestimmung rechtspraktisch in die Organstruktur einer monistisch verfassten Rechtsform zu implementieren. ${ }^{145}$ Diese Implementierung braucht nicht unbedingt mit einer materiellen Ausweitung der Arbeitnehmerrechte einherzugehen. Dies zeigt der Blick auf die SE: Zwar kann eine Arbeitnehmerbeteiligung bei einer monistisch verfassten Gesellschaft theoretisch mit einem Kompetenzzuwachs für die Arbeitnehmer einhergehen, da die Arbeitnehmervertreter dann nicht auf die Mitbestimmung

138 Eberspächer, ZIP 2008, 1951, 1956; Franzen, RdA 2004, 257, 263; Merkt, ZIP 2011, 1237, 1241; Raiser, Gutachten B zum 66. DJT Stuttgart 2006, B 109; Weiss/Seifert, ZGR 2009, 542, 579; ähnlich Braun, Die Sicherung der Unternehmensmitbestimmung im Lichte des europäischen Rechts, 2005, S. 192 f.

139 Eberspächer, ZIP 2008, 1951, 1956; vgl. Braun, Die Sicherung der Unternehmensmitbestimmung im Lichte des europäischen Rechts, 2005, S. 192 f.; Franzen, RdA $2004,257,263$.

140 So auch Weiss/Seifert, ZGR 2009, 542, 579; Franzen, RdA 2004, 257, 263.

141 Eberspächer, ZIP 2008, 1951, 1952; vgl. auch Teichmann, ZIP 2009, 1787, 1788.

142 Merkt, ZIP 2011, 1237, 1242.

143 EuGH v. 30.11.1995 - Rs. C-55/94, Slg. 1995, I-4165 - Gebhard.

144 Hübner, Kollisionsrechtliche Behandlung von Gesellschaften aus „nicht-privilegierten" Drittstaaten, 2010, S. 248 ff., $254 \mathrm{ff}$.

145 Vgl. Eidenmüller, ZIP 2002, 2233, 2242; W.-H. Roth, IPRax 2003, 117, 125; Zimmer, NJW 2003, 3585, 3591. 
im Aufsichtsrat - primär einem Kontrollorgan - beschränkt, sondern im Verwaltungsrat als dem Organ der unternehmerischen Oberleitung zu integrieren sind. ${ }^{146}$ Indem man den Arbeitnehmervertretern jedoch Posten nicht-geschäftsführender Board-Mitglieder zuweist und sie damit aus dem Tagesgeschäft heraushält und diese lediglich in solchen Ausschüssen gruppiert, die sich nicht mit der strategischen Unternehmensplanung, sondern primär mit Kontrollaufgaben befassen, lässt sich die Mitbestimmung auch in einer monistischen Auslandsgesellschaft auf dem bisher in Deutschland bestehenden Niveau eindämmen. ${ }^{147}$

Für eine Verhandlungslösung, die vor einer zwingenden Lösung als schonenderer Weg Vorrang haben müsste, ${ }^{148}$ spricht auch folgender Gedanke: Dem europäischen Richtliniengeber schien es in den Richtlinien 2001/86/EG und 2005/56/EG ausreichend, nur für den Fall des Scheiterns der Verhandlungen eine Auffangregelung zu schaffen. ${ }^{149}$ Europarechtlich ist es daher „folgerichtig", die Verhandlungslösung als weniger belastendes Konzept zur Implementierung der Mitbestimmung in Gesellschaften anzusehen. ${ }^{150}$ Da der EuGH in den Entscheidungen Inspire Art $^{151}$ und innoventif-Limited ${ }^{152}$ deutlich gemacht hat, den Gewährleistungsinhalt der primärrechtlichen Niederlassungsfreiheit unter Rekurs auf die Inhalte der sekundärrechtlichen Richtlinien zu bestimmen, erscheint es nahe liegend, dass er angesichts der in der Verschmelzungsrichtlinie 2005/56/EG zum Ausdruck kommenden Wertungen eine Sonderanknüpfung der Regeln zur unternehmerischen Arbeitnehmermitbestimmung an den inländischen Verwaltungssitz der Auslandsgesellschaft als gerechtfertigte Beschränkung der Niederlassungsfreiheit ansehen würde.

Die Erstreckung der Mitbestimmung auf Auslandsgesellschaften mit inländischem Verwaltungssitz lässt sich nach alledem unter Berücksichtigung einer Subsidiaritätsklausel sowie einer Verhandlungslösung europarechtskonform ausgestalten. ${ }^{153}$

146 Gruber/Weller, NZG 2003, 297, 298.

147 Gruber/Weller, NZG 2003, 297, 300 f.; vgl. auch Reichert/Brandes, ZGR 2003, 767, $795 \mathrm{f}$.

148 Sandrock, AG 2004, 57, 66; Raiser, Gutachten B zum 66. DJT Stuttgart 2006, B 109; Teichmann, ZIP 2009, 1787, 1788.

149 Henssler in GS Heinze, 2005, S. 333, 353

150 Henssler in GS Heinze, 2005, S. 333, 353

151 In der Inspire Art-Entscheidung hat der EuGH der Zweigniederlassungsrichtlinie einen abschließenden Charakter hinsichtlich der Bestimmung des Gewährleistungsinhalts der Niederlassungsfreiheit beigemessen, vgl. Ziemons, ZIP 2003, 1913, 1914 f. Vgl. etwa zur inhaltlichen Ausgestaltung der Niederlassungsfreiheit durch die Handelsvertreterrichtlinie EuGH v. 9.11.2000 - Rs. C-381/98, IPRax 2001, 225, 227, Tz. $20 \mathrm{ff}$. - Ingmar.

152 EuGH v. 1.6.2006 - Rs. C-453/04, Slg. 2006, I-4929 (Tenor).

153 Ebenso Kindler in MünchKomm. BGB, 5. Aufl. 2010, Int. GesR, Rz. 567 f.; a. A. Eberspächer, ZIP 2008, 1951, 1954 ff. 


\section{F. Zusammenfassung}

(1) Die paritätische Unternehmensmitbestimmung steht in der Kritik. Sie gilt als Nachteil im internationalen Wettbewerb der Rechtsformen; ferner soll sie wegen ihrer stakeholder-Orientierung die Kontrollfunktion des Aufsichtsrates beeinträchtigen. Es kann dahingestellt bleiben, ob diese Kritik trägt; Fakt ist jedenfalls, dass auf Grundlage der EuGH-Trilogie Centros, Überseering und Inspire Art immer mehr Unternehmen aus der Mitbestimmung "flüchten“, indem sie für ihre Inlandsaktivitäten anstelle einer deutschen Gesellschaftsform eine ausländische Rechtsform einsetzen.

(2) Die Unternehmensmitbestimmung ist gesellschaftsrechtlich zu qualifizieren. Sie findet daher auf Gesellschaften mit ausländischem Gesellschaftsstatut keine Anwendung. Ein ausländisches Gesellschaftsstatut erkennt die deutsche Rechtsordnung freilich nur bei Gesellschaften aus anderen EU-Mitgliedstaaten sowie aus den USA an, nicht jedoch bei Gesellschaften aus nicht privilegierten Drittstaaten, z. B. der Schweiz.

(3) Vor diesem Hintergrund wird diskutiert, das MitbestG 1976 de lege ferenda qua Legislativakt auf Auslandsgesellschaften mit Verwaltungssitz im Inland zu erstrecken. Ein solches Erstreckungsgesetz wäre indes nur dann systemkohärent, wenn es kumulativ den Anforderungen des IPR und des Europarechts genügen würde.

(4) Kollisionsrechtlich kommt die Durchsetzung des Mitbestimmungs-Erstreckungsgesetzes gegen ein ausländisches Gesellschaftsstatut (nur) mittels einer Sonderanknüpfung in Frage; der ordre public (Art. 6 EGBGB) ist zu diesem Zweck wegen seiner rein negativen Funktion ungeeignet. Die auf Wengler zurückgehende Lehre von der Sonderanknüpfung setzt erstens eine international zwingende Bestimmung (Eingriffsnorm) und zweitens einen hinreichenden Bezug des Sachverhalts zum Inland voraus.

(5) Eine Eingriffsnorm zeichnet sich dadurch aus, dass sie nicht nur den Interessenausgleich zwischen Privaten regelt, sondern auch öffentliche Interessen verfolgt. Das MitbestG 1976 geht über einen bloßen Interessenausgleich zwischen Privaten deutlich hinaus; es zielt laut Bundesverfassungsgericht maßgeblich auf die Verwirklichung sozialpolitischer und gesellschaftlicher Ziele und ist damit eine Eingriffsnorm. Sofern die Auslandsgesellschaft einen hinreichenden Bezug zum Inland hat, etwa aufgrund eines in Deutschland belegenen Verwaltungssitzes, ließe sich das MitbestG 1976 daher kollisionsrechtlich systemkohärent auf sie anwenden.

(6) Europarechtlich muss sich die Erstreckung der Unternehmensmitbestimmung auf Auslandsgesellschaften an der Niederlassungsfreiheit messen lassen, die grundsätzlich jegliche Beschränkung des Marktzugangs verbietet (Art. 49, 54 AEUV). Eine solche Beschränkung stellt auch die Mitbestimmung dar.

(7) Allerdings kann die Anwendung der Unternehmensmitbestimmung auf Auslandsgesellschaften unter gewissen Voraussetzungen im Sinne der Cassis de Dijon-/Gebhard-Formel des EuGH gerechtfertigt werden. So stellt der Arbeit- 
nehmerschutz einen zwingenden Grund des Allgemeininteresses dar. Die Unternehmensmitbestimmung ist ferner geeignet, dem Schutz der Arbeitnehmer zu dienen.

(8) Um der Schranken-Schranke der Erforderlichkeit zu genügen, müsste ein Mitbestimmungs-Erstreckungsgesetz allerdings eine Subsidiaritätsklausel enthalten, wonach das deutsche Mitbestimmungsregime zurücktritt, wenn das ausländische Gesellschaftsstatut bereits ein vergleichbares Mitbestimmungsniveau vorsieht. Ferner müsste ein Erstreckungsgesetz einer Verhandlungslösung insbesondere im Hinblick auf monistische Gesellschaften Vorrang vor einem zwingenden Auffangregime einräumen. Unter Berücksichtigung dieser beiden Einschränkungen ließe sich ein Mitbestimmungs-Erstreckungsgesetz jedoch entgegen zahlreicher Stimmen in der Literatur auch europarechtskonform ausgestalten. 